\title{
Molecular phylogeny of Malagasy reed frogs, Heterixalus, and the relative performance of bioacoustics and color-patterns for resolving their systematics
}

\author{
Katharina C. Wollenberg ${ }^{\mathrm{a}, *}$, Frank Glaw ${ }^{\mathrm{b}}$, Axel Meyer ${ }^{\mathrm{c}}$, Miguel Vences ${ }^{\mathrm{a}}$ \\ ${ }^{a}$ Zoological Institute, Division of Evolutionary Biology, Technical University of Braunschweig, Spielmannstraße 8, 38106 Braunschweig, Germany \\ ${ }^{\mathrm{b}}$ Zoologische Staatssammlung, Münchhausenstraße 21, 81247 München, Germany \\ ${ }^{\mathrm{c}}$ Lehrstuhl für Zoologie/Evolutionsbiologie, Department of Biology, University of Konstanz, 78457 Konstanz, Germany
}

\begin{abstract}
The members of the genus Heterixalus constitute one of the endemic frog radiations in Madagascar. Here we present a complete spe cies level phylogeny based on DNA sequences (4876 base pairs) of three nuclear and four mitochondrial markers to clarify the phylo genetic relationships among and within all known species of this genus, as well as the phylogenetic position of the monospecific Seychellean Tachycnemis seychellensis. Although the performance to resolve supported clades of Heterixalus species differed among the investigated gene fragments when analyzed separately, we could identify five well supported species groups within Heterixalus in the combined analysis of all gene fragments. Our data strongly support a Heterixalus Tachycnemis clade, and indicate the probable monophyly of Heterixalus placed sister to Tachycnemis. However, the diversification of these lineages may have happened in a short interval of time, leading to an unstable placement of Tachycnemis in the single gene fragment phylogenies. Referring to the hitherto exist ing classification of Heterixalus, which is predominantly based on chromatic and bioacoustic characters, we examined the relative per formance of these data sets relative to our molecular phylogeny. A Bayesian tree reconstructed with a bioacoustic data set yielded a higher resemblance to the molecular phylogeny than a tree constructed using a chromatic data set, which supports the importance of bioacoustic characters for systematic analyses of these anurans.
\end{abstract}

Keywords: Amphibia: Anura: Hyperoliidae: Heterixalus; Madagascar; Molecular phylogeny; Systematics; Color patterns; Bioacoustics

\section{Introduction}

The treefrog family Hyperoliidae is composed of 18 genera (Frost et al., 2006; AmphibiaWeb, 2006), all but two of which are endemic to Africa. The two exceptions are Heterixalus, endemic to Madagascar and containing 11 species, and the monotypic genus Tachycnemis, with the only species $T$. seychellensis being endemic to the Seychelles islands (Drewes, 1984). Although morphological phylogenies placed Tachycnemis as a basal representative of the family (Drewes, 1984; Channing, 1989),

\footnotetext{
* Corresponding author. Fax: +495313918198.

E mail address: kc.wollenberg@tu bs.de (K.C. Wollenberg).
}

molecular phylogenetic data support close relationships between Tachycnemis and Heterixalus, and the Heterixalus Tachycnemis clade being deeply nested within African hyperoliid genera, possibly sister to Afrixalus (Richards and Moore, 1996; Vences et al., 2003b; Frost et al., 2006). Molecular clock analyses indicated a relatively young divergence between African and Malagasy Seychellean hyperoliids at about 30 million years ago, much more recent than the Gondwanan connection between these land masses that was severed about 160130 mya (Briggs, 2003). This indicates that colonization of Madagascar and of India by these frogs must have occurred by overseas dispersal during the Cenozoic (Vences et al., 2003c). 
Despite the obvious biogeographic interest in understanding the relationships of the Malagasy Seychellean hyperoliid radiation, a well-resolved phylogeny using multiple markers and a dense taxon sampling is so far missing for this group. Their systematics was first established based on morphology and color (Blommers-Schlösser, 1982; Blommers-Schlösser and Blanc, 1991), and later refined with bioacoustic analyses (Glaw and Vences, 1993). Based on these data, three species groups are recognized (Glaw and Vences, 1993): (i) the H. betsileo group with $H$. betsi$l e o$, (ii) the $H$. tricolor group with $H$. tricolor, $H$. variabilis and $H$. andrakata, and (iii) the $H$. madagascariensis group, containing $H$. madagascariensis and $H$. boettgeri. At the time of the study of Glaw and Vences (1993), the calls of $H$. rutenbergi and $H$. alboguttatus were not known yet, $H$. carbonei and $H$. punctatus were only subsequently described, and $H$. luteostriatus was not yet considered a valid species (Vences et al., 2000). Here we present a molecular phylogeny of all known Heterixalus species and of Tachycnemis based on an analysis of four mitochondrial and three nuclear gene fragments. The two major goals in this study were: (1) to achieve a better understanding of the relationships and of the genetic differentiation within Heterixalus as a contribution to a future taxonomic revision of these frogs, and (2) to obtain molecular evidence for the monophyly or non-monophyly of Heterixalus with respect to Tachycnemis.

Anurans are known to have a high level of morphological similarity and, often, homoplasy (e.g., Emerson, 1986) that has substantially obscured their systematics before the advent of molecular characters (e.g., Bossuyt and Milinkovitch, 2000; Van der Meijden et al., 2005; Frost et al., 2006). Compared to most other anurans, hyperoliids of several genera show even lower levels of morphological divergence, and taxonomy in the past has therefore largely been based on color-patterns. In Heterixalus, Glaw and Vences (1993) considered morphometrics as not very informative for distinguishing Heterixalus species, and restricted taxonomically informative characters to body size, advertisement calls, and color. However, color-pattern is known to be highly polymorphic within species of other hyperoliid genera, e.g., Hyperolius (Schiøtz, 1999; Amiet, 2004). Furthermore, the color-patterns in some species undergo permanent ontogenetic color changes (Richards, 1982). They are known to be under hormonal control (e.g., Hyperolius argus, Hayes and Menendez, 1999), which is related to sexual dimorphism. Intraspecific and intersexual color-pattern polymorphism also occurs in Heterixalus ( $H$. alboguttatus and H. variabilis, Glaw and Vences, 1994; Schmidt et al., 2001).

Color and/or pattern are known to be important for mate recognition or sexual selection in many taxa such as birds or in cichlid fishes (Salzburger and Meyer, 2004). Although in frogs there is little evidence for female choice based on male color-patterns, some indications for visual color-based mate recognition may exist in diurnal poison frogs (Summers et al., 2003). Sexual dichromatism is known from only 32 out of 225 polychromatic frogs (Hoffman and Blouin, 2000). Many of these are hyperoliids of the genera Hyperolius and Heterixalus, and the hypothesis can therefore not be ruled out that their coloration might be influenced by sexual selection. In species-level systematics, characters under sexual selection can be very useful in phylogenetically assigning specimens to biological species. In contrast, when the phylogenetic relationships among distantly related species are to be reconstructed, characters under strong selection (be it sexual or natural) are unsuitable because they are likely to show high levels of homoplasy. It furthermore has been suggested that in aposematic animals color-patterns tend to be in greater conflict with morphological signals concerning their phylogenetic significance than in non-aposematic animals (Areekul and Quicke, 2006). Although some Heterixalus have a rather vivid coloration, it is very unlikely that this constitutes an aposematic signal. Conclusive statements on the vivid and often dimorphic color of these frogs require further experimental testing.

In contrast, there is no doubt that the advertisement calls of frogs are the main mechanism of mate recognition and sexual selection in this amphibian group. Bioacoustic characters are of excellent use in taxonomy, although the rate in which different call parameters evolve can vary among groups (Ryan, 1986; Cocroft and Ryan, 1995; Richards, 2006) and their value for phylogenetic reconstruction is poorly understood.

In reference to the hitherto existing classification of Heterixalus, being predominantly based on chromatic and bioacoustic characters, a third goal of this study was to examine the performance of these characters to resolve the phylogenetic relationships among populations and species of Heterixalus as they were recovered using the molecular data sets. We use the molecular phylogeny as a basis of comparison to evaluate the systematic relevance of bioacoustic and chromatic characters for the identification of species and major clades within Heterixalus.

\section{Materials and methods}

We obtained DNA-sequences of 34 individuals from all currently valid 11 Heterixalus species and one Tachycnemis seychellensis (Electronic Supplement Table E1), with multiple populations from much of the known range sampled for most species. We analyzed one fragment of the mitochondrial 12S rRNA, Cytochrome $b$ (Cytb) and Cytochrome Oxidase Subunit 1 genes (COI), two fragments of the 16S rRNA gene, and one fragment each of the nuclear Tyrosinase, Rhodopsin and Rag-1 genes. In total, this yielded 4876 basepairs of sequence data (Table E2). The full alignment was submitted to TreeBase (http:// www.treebase.org; Sequences were submitted to GenBank (accession numbers EF646474 EF646728; Table E1, Benson et al., 2004). For primer information, see Table E2. PCR was performed according to the reaction conditions and thermocycling protocols described in Vences et al. 
(2004, 2003a). Sequencing reactions were performed with the forward primers by Macrogen Inc., Korea (cf. Vences et al., 2004). The obtained sequences were edited conservatively and verified as hyperoliid DNA via BLAST searches.

The phylogenetic analysis of the molecular data was performed for each gene separately, except for the $12 \mathrm{~S}$ and $16 \mathrm{~S}$ fragments, which were combined to an "rRNA" data set. Subsequently, all genes were analyzed in a combined data set in order to profit from the phylogenetic resolution provided in different parts of the tree by the separate data sets (Pereira et al., 2002; Heckman et al., 2007). Sequences of species of the hyloid genus Bufo as available from Genbank were used as outgroup. Sequences of other taxa (Leptodactylus fuscus, Mantidactylus (now Blommersia) sp. (Mayotte), Mantidactylus (now Blommersia) wittei, Astylosternus sp., Leptopelis natalensis, Hyperolius viridiflavus, Bufo sp., and Afrixalus delicatus; Vences et al., 2003c) were included as hierarchical outgroups. For the combined data set, heuristic searches were performed using PAUP* (v4b10; Swofford, 2001) under the criterium of maximum parsimony (MP), with 2000 non-parametric bootstrap replicates (Felsenstein, 1985). Characters in MP searches were treated as unordered with equal weight. Gaps were treated as "missing"; multistate characters were interpreted as "uncertain". Trees were computed with random stepwise addition of taxa, and branch swapping was performed with the TBR (Tree-Bisection-Reconnection) algorithm. Except for the rRNA data set, MP bootstrap searches could not be performed for the single-gene fragment data sets due to the low amount of variation among several terminal taxa, and a consequently large number of equally parsimonious trees. Maximum likelihood (ML) phylogenies of all molecular data sets were established using PhyML (Guindon and Gascuel, 2003), on the basis of substitution models selected by ModelGenerator using the Akaike Information Criterion AIC2 (Posada and Buckley, 2004; Keane et al., 2006, Table E3) with 2000 bootstrap replicates. As genetic distances of the sequenced 16S rRNA gene fragment 1 have already been reported for a variety of amphibian species and this marker has been proposed as suitable for DNA barcoding of these organisms (Vences et al., 2005), we computed a genetic distance matrix for all specimens for a later comparison of interspecific and intraspecific variation. We furthermore computed Bayesian phylogenies using $\mathrm{MrBa}-$ yes (v 3.1.2; Huelsenbeck and Ronquist, 2001) for all single-gene fragment data sets and for the combined data set. For the combined data set, we defined each of the $12 \mathrm{~S}$ rRNA and 16S rRNA fragments, and in the coding gene fragments each codon position, as separate partitions (Table E4). For all partitions, we computed best-fit models of evolution using MrMODELTEST under the AIC (Nylander, 2004, Table E4). These were implemented in the Bayesian analysis, which was then run under both the maximum (17 partitions in total) and the minimum partitioning strategies ( 7 partitions in total Table E4). Two million generations were run, every 100th tree sampled, and the first 10,000 generations discarded. Our preferred molecular phylogeny was the MrBayes topology obtained under the maximum partitioning strategy using the all-genes data set (depicted in Fig. 1). The single-gene bayesian phylogenies can be viewed in Fig. 2.

For testing the performance of bioacoustic versus chromatic characters in phylogenetic reconstruction relative to the preferred molecular phylogeny, chromatic and call data matrices were constructed on the basis of color-pattern and call observations allowing multistate characters (Table E5, E6). For the color data set, we coded traits such as skin color, "sun color" (reflective color in basking specimen), eye color, and certain pattern characteristics. Because of potential hormonal influences on hyperoliid coloration (Richards, 1982; Hayes and Menendez, 1999), we did not include absolute color values in the chromatic data matrix. For the acoustic data set, we recorded the presence versus absence of certain note types and their pulse rates (Table E5). No invariate characters were recorded. In the color-pattern data set, 7 of 14 recorded characters were polymorphic, while in the call data set none of the eight recorded characters was polymorphic (although more missing characters occurred here). These matrices were established for a limited set of Heterixalus populations, for which at least several specimens could be sampled for chromatic and bioacoustic data (adult males and females in daylight coloration, at least several males for bioacoustics). Tachycnemis seychellensis was used as outgroup. We computed phylogenies for both data sets using MrBayes. We subsequently compared the tree topologies established with the bioacoustic and chromatic data sets to the topology of our preferred molecular tree. Congruence between pairs of phylogenies was determined with the Software COMPONENT (v2.0, Page, 1993). For an objective measure of tree similarity we followed the approach in Page et al. (2002). We computed triplet tree comparison metrics (Day, 1986), which treat trees as a set of clusters (Day, 1985), and tree distance was computed according to the distances between these sets. Triplet comparison is recommended for rooted trees (Page, 1993). The resulting distance measures are based on counting how many triplets are resolved in each tree and whether they are resolved the same way. Dissimilarity values were calculated, such as the number of strict joint assertions (SJA), which is the number of resolved triplets different in the two trees divided by the number of triplets resolved in the two trees (Estabrook et al., 1985; Day, 1986; Page et al., 2002). These measures are the rooted equivalents of the measures for quartets described by Day (1986). Null distributions for these measures were obtained by computing the distances between 1000 pairs of random trees using COMPONENT (Page, 1993; Page et al., 2002). Furthermore, Consistency, Homoplasy and Retention Indices (Kluge and Farris, 1969; Farris, 1989), as well as the Rescaled Consistency Index (Farris, 1989), were computed with PAUP for the color-pattern, call and molecular phylogenies. 


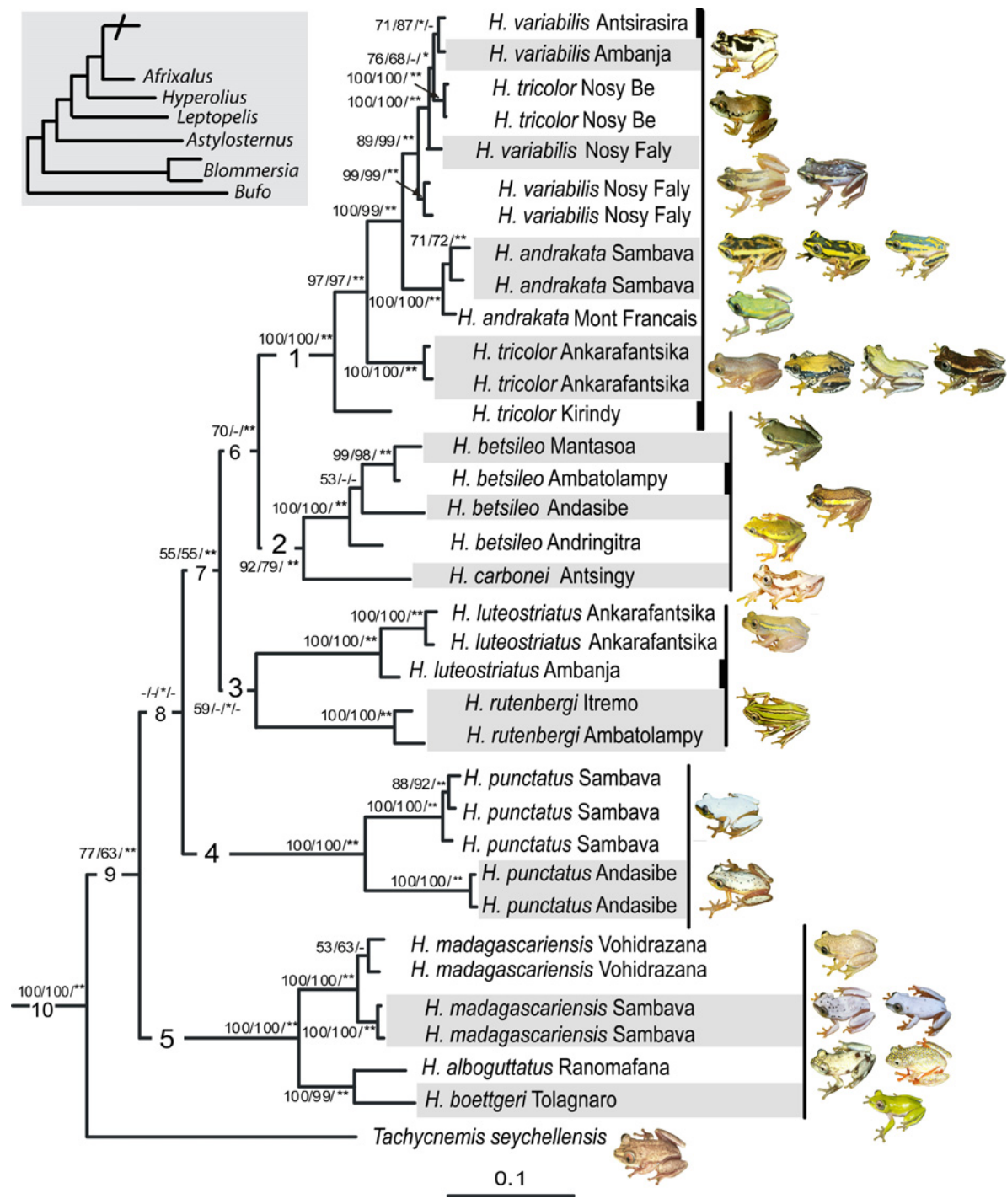

Fig. 1. Molecular phylogeny of all known Heterixalus species obtained by Bayesian inference under the maximum partitioning strategy, based on a total of 4876 base pairs from the concatenated set of 5 mitochondrial and 3 nuclear gene fragments. Node values indicate $>50 \%$ in ML phylogeny (first numbers), high bootstrap values $>50 \%$ in MP analysis (second numbers), and Bayesian posterior probabilities $>95 \%$ (asterisks) or $>99 \%$ (double asterisks). If the Bayesian posterior probabilities obtained under the minimum partitioning strategy differed from the values obtained under the maximum partitioning strategy, a fourth position is provided with asterisks $(>95 \%)$, double asterisks $(<99 \%)$ or " " $(<95 \%)$. ML and MP analyses yielded the same topology and are therefore not shown. Relationships among outgroup taxa are shown in the inset box. Numbers 15 within branches indicate the species groups proposed by us. Numbers 610 indicate deeper lineage splits (cf. Table 3). Images are sorted by species group. Populations for which no image is included are indicated by a small black bar, light gray stripes help to assort pictures to taxon names.

\section{Results and discussion}

\subsection{Molecular phylogeny of Heterixalus and Tachycnemis}

The phylogenetic analysis based on the multi-gene data set provided a tree (Fig. 1) in which five main clades were supported by high Bayesian posterior probabilities (>95\%, Table 3), and all but one of these clades also by MP and ML bootstrap values $>79 \%$. These are (1) a highly supported group of $H$. variabilis, $H$. tricolor, $H$. andrakata; (2) a clade including $H$. betsileo and $H$. carbonei; (3) a clade containing $H$. luteostriatus and $H$. rutenbergi; (4) a clade containing a single species, $H$. punctatus; and (5) a clade with $H$. madagascariensis, $H$. alboguttatus and $H$. boettgeri. The taxa in group 1 are distributed in northern Madagascar, groups 2 and 3 contain taxa from the highlands and western Madagascar, the species in clade 5 are allopatrically distributed along Madagascar's east coast, and $H$. punctatus (clade 4) is found in eastern and northeastern Madagascar. These results agree in part with the species groups defined by Glaw and Vences (1993). Our clade 1 corresponds to the $H$. tricolor group as defined in that revision, clade 2 corresponds to the $H$. betsileo group with the addition of $H$. carbonei (Vences et al., 2000), and clade 5 corresponds to the $H$. madagascariensis group of Glaw and Vences (1993), with additional inclusion of $H$. alboguttatus. However, a few relationships resolved by our analysis were unexpected, such as the placement of 

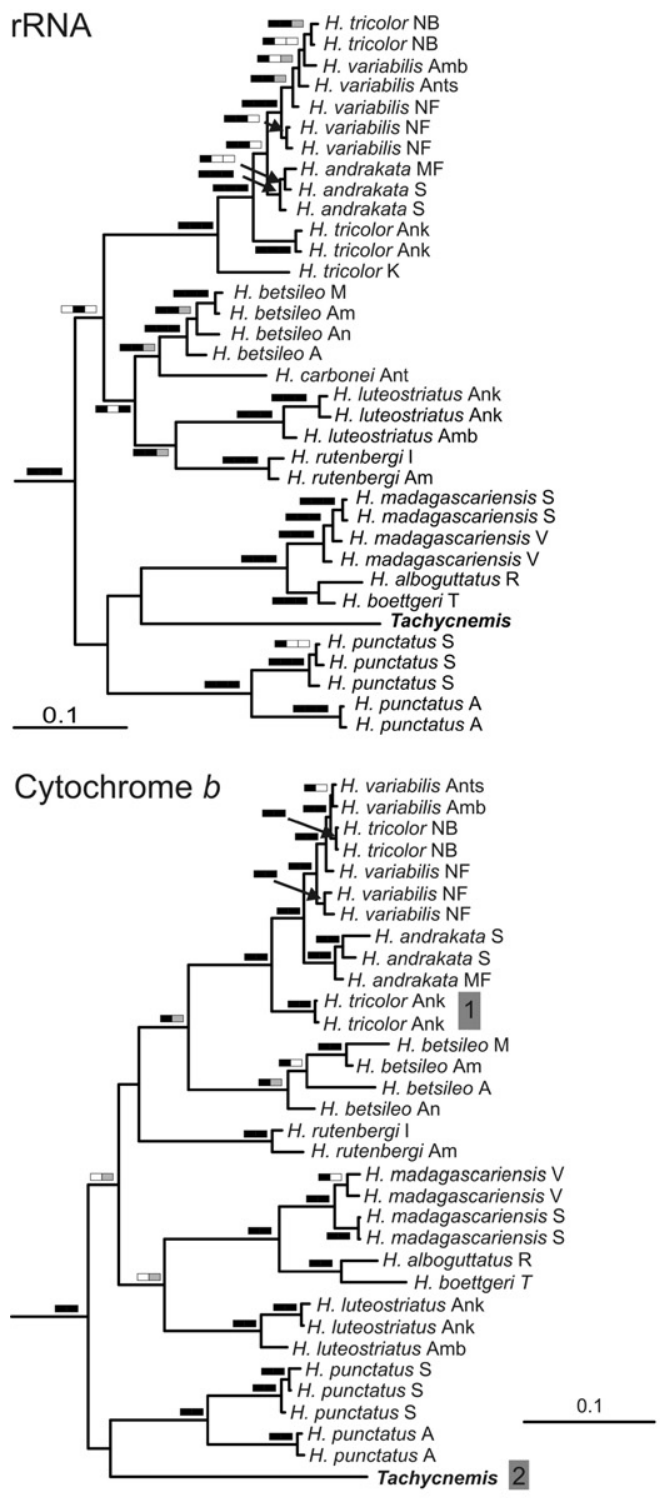

Cytochrome oxidase I

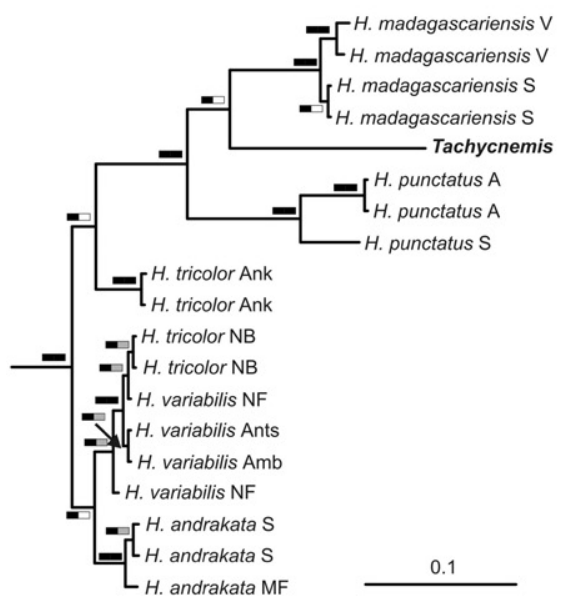

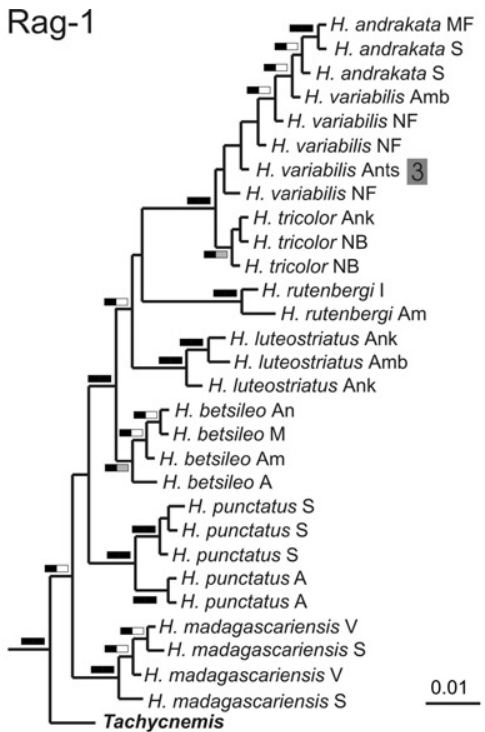
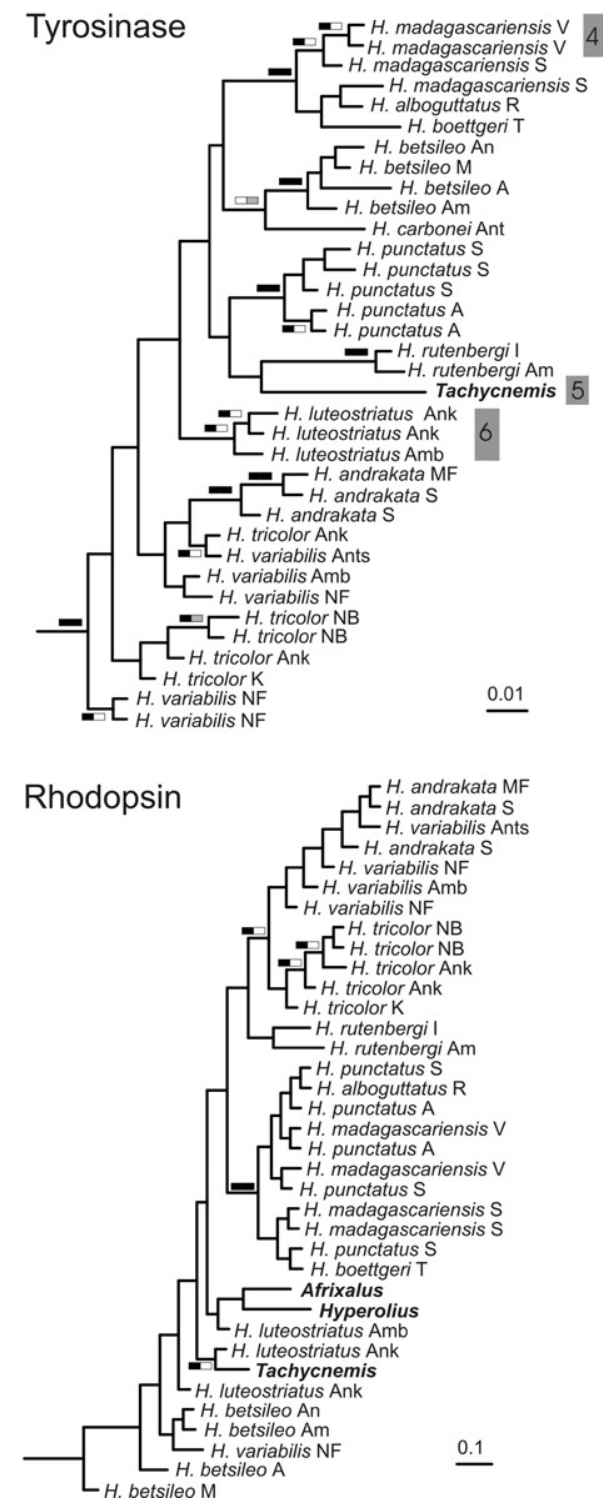
$H$. rutenbergi sister to $H$. luteostriatus which was however poorly supported by ML and MP analyses.

Our analysis revealed instances of very low genetic divergences among species, deep divergences within species, and mitochondrial paraphyly of species, indicating that the species-level classification of Heterixalus is in need of revision.

Very shallow divergences were found between Heterixalus tricolor from the type locality Nosy Be, H. variabilis and $H$. andrakata. This lack of differentiation (e.g., $0.57 \%$ $16 \mathrm{~S}$ sequence divergence between $H$. tricolor and $H$. variabilis from their respective type localities, Nosy Be and Nosy Faly and $0.76 \% 16 \mathrm{~S}$ sequence divergence between $H$. tricolor from Nosy Be and $H$. andrakata) would suggest to place $H$. andrakata, $H$. variabilis and $H$. tricolor from the type locality Nosy Be in a single species. This seems also to be supported by the fact that one of the three individuals of $H$. variabilis from Nosy Faly was placed into a different subclade (Fig. 1). However, considering that Glaw and Vences (1993) reported some, albeit small, call differences among these taxa, more data on their contact zones will be necessary for definitive taxonomic conclusions. Low divergences are also found between Heterixalus alboguttatus and $H$. boettgeri $(0.38 \% 16 \mathrm{~S}$ sequence divergence). From these allopatrically distributed species in south-eastern Madagascar only single individuals were studied here.

Deeply divergent genetic lineages were found (1) in Heterixalus tricolor (up to $2.5 \% 16 \mathrm{~S}$ sequence divergence between populations), making this species paraphyletic with respect to $H$. andrakata and $H$. variabilis, and (2) in $H$. punctatus $(3.8 \% 16 \mathrm{~S}$ sequence divergence between the two studied populations). The divergent populations of $H$. tricolor occur in arid western Madagascar, where suitable habitat may be patchy and populations could be historically strongly isolated and fragmented. The two $H$. punctatus populations studied, Sambava and Andasibe, are located in north-eastern and central eastern Madagascar. A denser sampling of intervening populations will be necessary to understand the taxonomic significance of their genetic differentiation.

In contrast to the five clades within Heterixalus, the more basal phylogenetic relationships were only partially resolved in our tree, depending on the method of phylogenetic investigation (Figs. 1 and 2). Although the clade containing all Heterixalus plus Tachycnemis received maximum support in all analyses, the monophyly of Heterixalus could not be unambiguously clarified. Our preferred Bayesian phylogeny (Fig. 1) placed Tachycnemis seychellensis basal to a clade of all Heterixalus, with high statistical support. MP and ML analyses also supported this placement, though not with maximum values. In the single-gene phylogenies, the position of Tachycnemis varied. The reason for this may partially be due to missing sequence data for some Heterixalus populations. Furthermore, the performances of the nuclear Rhodopsin and Tyrosinase fragments for reconstructing relationships among closely related amphibian species have not been subject to investigation yet. Assuming a relatively large ancestral population size, and taking into account the relatively young age of the hyperoliid radiation in Madagascar and the Seychelles, incomplete lineage sorting may be responsible for the partially different topologies obtained with these markers (Maddison, 1997; Heckman et al., 2007).

Assuming the overseas dispersal scenario of Vences et al. (2003c), and not taking into account the unlikely scenario of earlier, now extinct hyperoliid radiations on Madagascar, our results indicate that the ancestor of Tachycnemis (1) either dispersed from Madagascar to the Seychelles shortly after having arrived in Madagascar, and just before the diversification of the (monophyletic) Heterixalus started, or (2) dispersed after the basal clades of Heterixalus originated in Madagascar, which could imply paraphyly of Heterixalus.

\subsection{Phylogenetic performance of bioacoustic and chromatic characters}

In the color-pattern based phylogeny, most populations of the a-priori defined Heterixalus species were placed in monophyletic groups. This is not surprising since the prevalent color-pattern similarity determined the original taxonomic association of individuals to species (e.g., Blommers-Schlösser, 1982; Glaw and Vences, 1993). An exception was Heterixalus betsileo; the Ambatolampy population of this species was placed with $H$. boettgeri. Both share a largely uniform vivid green color. The phenotypic convergence of these two species spans all 14 investigated color and pattern traits, except the fact that $H$. betsileo from Ambatolampy is polymorphic concerning eye coloration (chromatic character 4, Table E5). They possess the smallest number of derived chromatic character states after $T$. seychellensis (only longitudinal stripes on shanks are present in both), and therefore they are placed basal to all other Heterixalus populations.

The two $H$. tricolor populations studied for coloration were placed in one clade in the color-pattern based tree, contrasting to the molecular analysis. The color-pattern

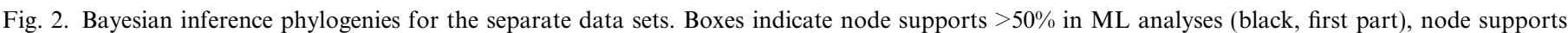

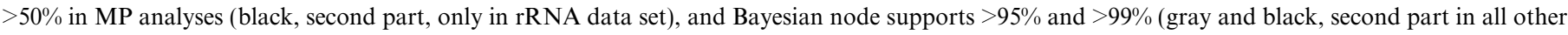

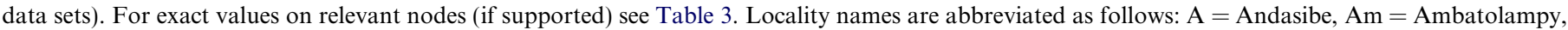

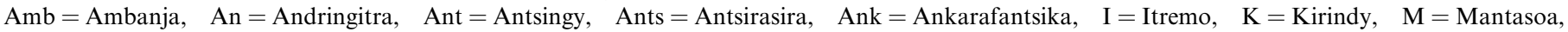

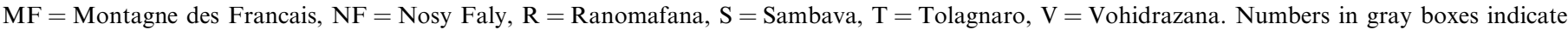

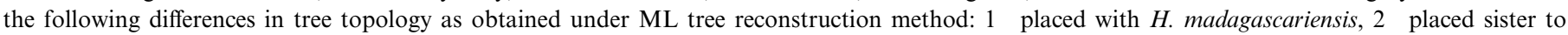
Heterixalus, 3 placed with H. tricolor, 4 placed with other H. madagascariensis, 5 placed sister to Heterixalus, 6 placed in H. tricolor group. 

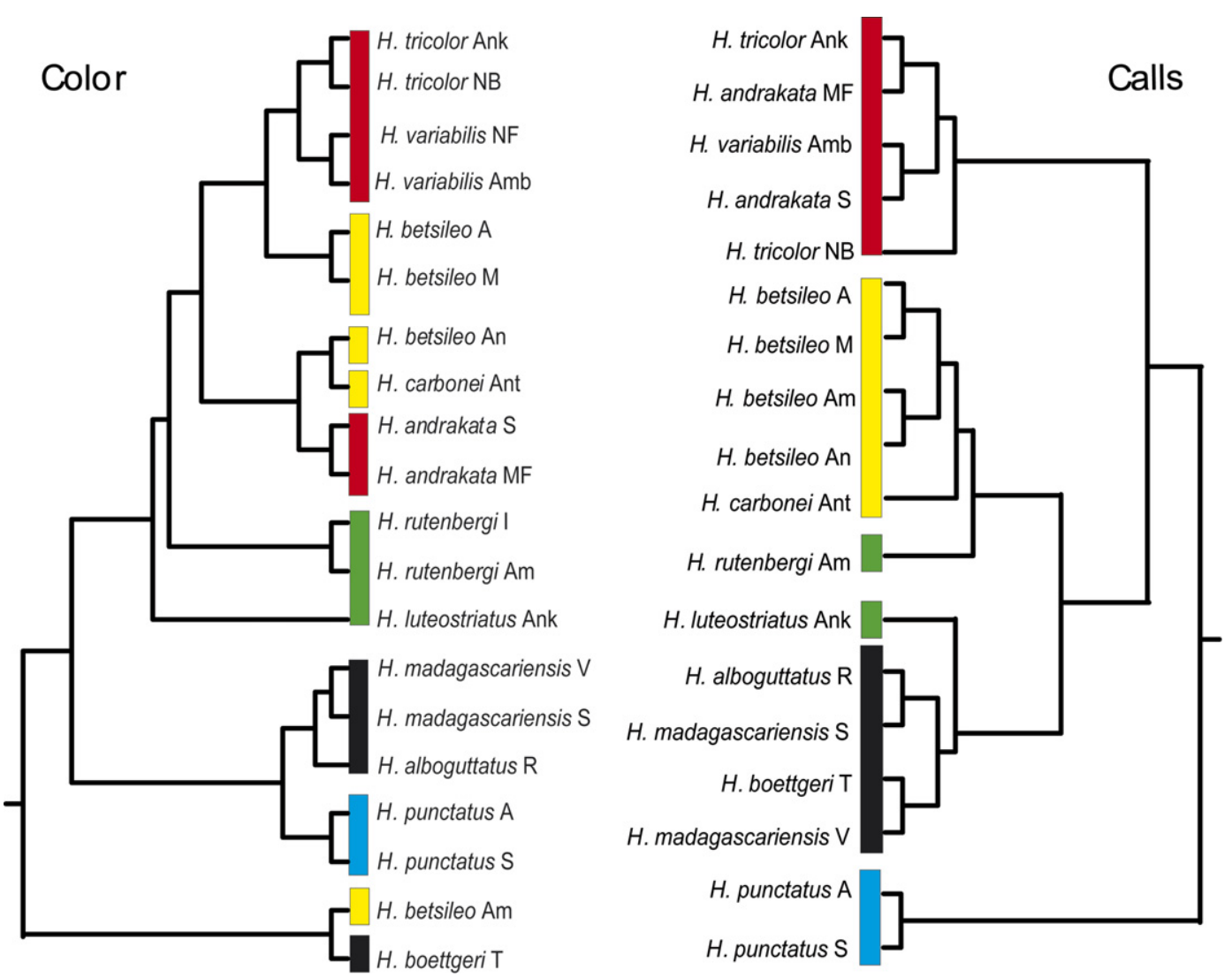

Fig. 3. Bayesian inference phylogenies of Heterixalus species according to the chromatic (left) and bioacoustic (right) data sets. Colored boxes represent molecular clades: red $=1$, yellow $=2$, green $=3$, blue $=4$, black $=5$. Locality names are abbreviated as follows: $\mathrm{A}=\mathrm{Andasibe}, \mathrm{Am}=\mathrm{Ambatolampy}$, $\mathrm{Amb}=$ Ambanja, $\mathrm{An}=$ Andringitra, Ant $=$ Antsingy, Ank $=$ Ankarafantsika, $\mathrm{I}=$ Itremo, $\mathrm{M}=$ Mantasoa, $\mathrm{MF}=\mathrm{Montagne}$ des Francais, $\mathrm{NF}=\mathrm{Nosy}$ Faly, $\mathrm{R}=$ Ranomafana, $\mathrm{S}=$ Sambava, $\mathrm{T}=$ Tolagnaro, $\mathrm{V}=$ Vohidrazana. Tachycnemis seychellensis was used as outgroup (not shown). For definition of characters and character matrices, see electronic supplementary material. (For interpretation of the references to the color in this figure legend the reader is referred to the web version of this article.)

Table 1

Indices for the degree of homoplasy in the respective phylogenies

\begin{tabular}{lllll}
\hline & CI & HI & RI & RC \\
\hline Molecular phylogeny & 0.59 & 0.10 & 0.81 & 0.47 \\
Color phylogeny & 0.55 & 0.45 & 0.76 & 0.41 \\
Call phylogeny & 0.90 & 0.10 & 0.96 & 0.87 \\
\hline
\end{tabular}

A Rescaled Consistency Index $=1$ would indicate that explaining the tree topology does not require any reversals or parallelisms.

based tree furthermore did not recover any of the major clades identified by the molecular data (except clade 5 that is composed of a single species), suggesting a rather limited value of chromatic characters for Heterixalus systematics.
In contrast, the bioacoustic data set was able to recover four of the five major clades suggested by the molecular analysis (clades 1, 2, 4, 5), although this analysis (Fig. 3) was based on only eight characters. In the call-based tree, three a-priori species were not recovered as monophyletic groups: $H$. madagascariensis, $H$. tricolor and $H$. andrakata. Populations of $H$. madagascariensis clearly form a monophyletic group in the molecular analysis, and $H$. andrakata populations are a molecular clade too, although with poor support. At the level of closely related (allopatric) species of Heterixalus, call data may confound lineages which probably have just not diverged enough bioacoustically.

In concert with these general observations, homoplasy indices were higher in the chromatic data set than in the

Table 2

Dissimilarity values for the comparisons of color and call phylogenies with the Bayesian molecular phylogeny

\begin{tabular}{lllllllll}
\hline Tree1 & Tree2 & SD & EA & DC & SJA & $Q$ & $s$ \\
\hline Molecular & Color & 0.289 & 0.298 & 0.271 & 0.279 & 1330 & 933 \\
Molecular & Calls & 0.341 & 0.341 & 0.341 & 0.341 & 969 & 639 \\
\hline
\end{tabular}

$\mathrm{SD}=$ symmetric difference, $\mathrm{EA}=$ explicitly agree, $\mathrm{DC}=$ do not conflict, $\mathrm{SJA}=$ strict joint assertions, $Q=$ maximum possible resolved, $s=$ resolved and same, $d=$ resolved and different. A SJA value $=1$ would mean that all triplets differ among the compared topologies. Distances between 1000 random trees with 21 taxa yielded a $\mathrm{SJA}=0.667$ with standard error $=0.644$. 
Table 3

Node supports (node numbers refer to Fig. 1) for the various data sets/phylogeny reconstruction methods

\begin{tabular}{|c|c|c|c|c|c|c|c|}
\hline Node & All genes & rRNA & Cytb & COI & Rag 1 & Tyr & Rhod \\
\hline 1 & $100 / 100 / * *$ & $100 / 100 / * *$ & $99 / * *$ & / & $100 / * *$ & / & $60 /$ \\
\hline 2 & $92 / 79 / * *$ & $91 / 58 / *$ & $99 / * *$ & Missing data & $70 / * *$ & $/ *$ & / \\
\hline 3 & $59 / / * /$ & $87 / 64 / *$ & /X & Missing data & $\mathrm{X} / \mathrm{X}$ & / & / \\
\hline 4 & $100 / 100 / * *$ & $100 / 100 / * *$ & $100 / * *$ & $100 / * *$ & $98 / * *$ & $72 / * *$ & $\mathrm{X} / \mathrm{X}$ \\
\hline 5 & $100 / 100 / * *$ & $100 / 100 / * *$ & $100 / * *$ & $100 / * *$ & $99 / * *$ & $59 / * *$ & $\mathrm{X} / \mathrm{X}$ \\
\hline 6 & $70 / / * *$ & $/ 51 /$ & $57 / *$ & / & / & / & / \\
\hline 7 & $55 / 55 / * *$ & $\mathrm{X} / \mathrm{X}$ & / & Missing data & $65 / * *$ & / & / \\
\hline 8 & $/ / * /$ & / / & / & Missing data & / & / & / \\
\hline 9 & $77 / 63 / * *$ & / / & / & Missing data & $79 /$ (89) & / & $\mathrm{X} /$ \\
\hline 10 & $100 / 100 / * *$ & $99 / 100 / * *$ & $89 / * *$ & / & $99 / * *$ & / & / \\
\hline
\end{tabular}

Node supports are written in the order $>50 \%$ in ML analysis, $>50 \%$ in MP analysis (only in "all genes" and rRNA data sets) $>95 \%$ in Bayesian analysis (maximum partitioning strategy) and $>95 \%$ in Bayesian analysis (minimum partitioning strategy) Asterisks in Bayesian analysis indicate a node support of $9598 \%$, and double asterisks indicate a node support of $99100 \%$. " " indicates low node support; "X" indicates strong node support for a constellation different from the one in Fig. 1. Node 9 equals the phylogenetic position of Tachycnemis, node 10 equals the monophyly of the Heterixalus/Tachycnemis clade.

call data set (Table 1). The "dissimilarity values" among pairs of tree topologies (Estabrook et al., 1985; Day, 1986) as summarized in Table 2 were more ambiguous. Both color-pattern and call phylogenies were more similar to the molecular phylogeny than expected by random, and the similarity between call and molecular phylogenies was slightly higher than that between color and molecular phylogenies $(\triangle \mathrm{SJA} \quad 0.062$, Table 2$)$. The indices measuring triplet differences (SD and SJA) were unexpectedly higher in the molecular-to-call data set comparison than in the molecular-to-chromatic data set comparison, but the measures for agreeing or not conflicting triplets were higher in the call-to-molecular data set comparison than in the colorto-molecular data set comparison. An explanation for this might be that the used test's sensitivity is not very high triplet measures are generally considered as having a poor resolution (Penny and Hendy, 1985). However, altogether the triplet comparison tests largely supported a better agreement between the call and the molecular data set as compared to the color-to-molecular data sets. Our results therefore confirm previous assumptions (Glaw and Vences, 1993; Vences et al., 2000) that bioacoustics perform better in species-level systematics of these frogs than chromatic characters.

\section{Acknowledgments}

We are grateful to F. Andreone, L. Raharivololoniaina and D.R. Vieites for assistance in the field and to M. Kondermann and I. Somorjai who contributed significantly to the lab work. Field work was made possible through an agreement of collaboration with the Département de Biologie Animale, Université d'Antananarivo, Madagascar. We thank O. Ramilijaona and N. Raminosoa for continued support, and the Madagascan "Ministère de l'Environnement, des Eaux et Forêts", as well as the "Association Nationale pour la Gestion des Aires Protegées", for issuing research permits.

\section{Appendix A. Supplementary data}

Supplementary data associated with this article can be found, in the online version, at doi:10.1016/j.ympev. 2007.06.024.

\section{References}

Amiet, J. L., 2004. Une nouvelle espèce d' Hyperolius du Cameroun (Amphibia, Anura, Hyperoliidae). Rev. Suisse Zool. 111, 567583.

AmphibiaWeb (Accessed June, 2006). Information on amphibian biology and conservation (web application). AmphibiaWeb, Berkeley, California.

Areekul, B.J., Quicke, D.J., 2006. The use of color characters in phylogenetic reconstruction. Biol. J. Linn. Soc. Lond. 88, 193202.

Benson, D.A., Karsch Mitzrachi, I., Lippman, D.J., Ostell, J., Wheeler, D.L., 2004. GenBank: update. Nucl. Acids Res. 32, D23 D26, Database Issue.

Blommers Schlosser, R.M.A., 1982. Observations on the Malagasy frog genus Heterixalus Laurent, 1944 (Hyperoliidae). Beaufortia 32, 111.

Blommers Schlosser, R.M.A., Blanc, C.P., 1991. Amphibiens (première partie). Faune de Madagascar 75, 1379.

Bossuyt, F., Milinkovitch, M.C., 2000. Convergent adaptive radiations in Madagascan and Asian ranid frogs reveal covariation between larval and adult traits. Proc. Natl. Acad. Sci. USA 97, 65856590.

Briggs, J.C., 2003. The biogeographic and tectonic history of India. J. Biogeogr. 30, 381388.

Channing, A., 1989. A re evaluation of the phylogeny of Old World treefrogs. S. Afr. J. Zool. 24, 116131.

Cocroft, R.B., Ryan, M.J., 1995. Patterns of advertisement call evolution in toads and chorus frogs. Anim. Behav. 49, 283303.

Day, W.H.E., 1985. Optimal algorithms for comparing trees with labeled leaves. J. Classif. 1, 728 .

Day, W.H.E., 1986. Analysis of quartet dissimilarity measures between undirected phylogenetic trees. Syst. Zool. 35, 325333.

Drewes, R.C., 1984. A phylogenetic analysis of the Hyperoliidae (Anura): treefrogs of Africa, Madagascar and the Seychelles Islands. Proc. Calif. Acad. Sci. 139, 170.

Emerson, S.B., 1986. Convergence and morphological constraint in frogs: variation in postcranial morphology. Field. Zool. 43, 119.

Estabrook, G.F., McMorris, F.R., Meacham, C.A., 1985. Comparison of undirected phylogenetic trees based on subtrees of four evolutionary units. Syst. Zool. 34, 193200.

Farris, J.S., 1989. The retention index and the rescaled consistency index. Cladistics 5, 417419. 
Felsenstein, J., 1985. Phylogenies and the comparative method. Am. Nat. $125,115$.

Frost, D.R., Grant, T., Faivovich, J., Bain, R., Haas, A., Haddad, C.F.B., De Sá, R.O., Channing, A., Wilkinson, M., Donnellan, S.C., Raxworthy, C.J., Campbell, J.A., Blotto, B.L., Moler, P., Drewes, R.C., Nussbaum, R.A., Lynch, J.D., Green, D.M., Wheeler, W.C., 2006. The amphibian tree of life. Bull. Am. Mus. Nat. Hist. 297, 1371.

Glaw, F., Vences, M., 1993. Zur Bioakustik, Biologie und Systematik der Gattung Heterixalus aus Madagaskar (Anura: Hyperoliidae). Salam andra 29, 212230.

Glaw, F., Vences, M., 1994. A fieldguide to the amphibians and reptiles of Madagascar. Second edition, including mammals and freshwater fish. Koln, Vences \& Glaw, 480 pp.

Guindon, S., Gascuel, O., 2003. A simple, fast, and accurate algorithm to estimate large phylogenies by Maximum Likelihood. Syst. Biol. 52, 696704.

Hayes, T.B., Menendez, K.P., 1999. The effect of sex steroids on primary and secondary sex differentiation in the sexually dichromatic reedfrog (Hyperolius argus: Hyperoliidae) from the Arabuko Sokoke forest of Kenya. Gen. Comp. Endocrinol. 115, 188199.

Heckman, K.L., Mariani, C.L., Rasoloarison, R., Yoder, A., 2007. Multiple nuclear loci reveal patterns of incomplete lineage sorting and complex species history within western mouse lemurs (Microcebus). Mol. Phylogenet. Evol. 43, 353367.

Hoffman, E.A., Blouin, M.S., 2000. A review of color and pattern polymorphism in anurans. Biol. J. Linn. Soc. 70, 633665.

Huelsenbeck, J.P., Ronquist, F., 2001. MRBAYES: Bayesian inference of phylogenetic trees. Bioinformatics 17, 754755.

Keane, T.M., Creevey, C.J., Pentony, M.M., Naughton, T.J., McInerney, J.O., 2006. Assessment of methods for amino acid matrix selection and their use on empirical data shows that ad hoc assumptions for choice of matrix are not justified. BMC Evol. Biol. 6, 29.

Kluge, A.G., Farris, J.S., 1969. Quantitative phyletics and the phylogeny of the anurans. Syst. Zool. 18, 132.

Maddison, W.P., 1997. Gene trees in species trees. Syst. Biol. 46, 523536.

Nylander, J.A.A., 2004. MrModeltest v2. Program distributed by the author. Evolutionary Biology Centre, Uppsala University.

Page, R.D.M., 1993. COMPONENT, Tree Comparison Software for Microsoft ${ }^{\circledR}$ Windows ${ }^{\mathrm{TM}}$. The Natural History Museum, London.

Page, R.D.M., Cruickshank, R., Johnson, K.P., 2002. Louse (Insecta: Phthiraptera) mitochondrial $12 \mathrm{~S}$ rRNA secondary structure is highly variable. Insect Mol. Biol. 11, 361369.

Pereira, S.L., Baker, A.J., Wajntal, A., 2002. Combined nuclear and mitochondrial DNA sequences resolve generic relationships within the Cracidae (Galliformes, Aves). Syst. Biol. 51, 946958.

Penny, D., Hendy, M.D., 1985. The use of tree comparison metrics. Syst. Zool. 34, 7582.

Posada, D., Buckley, T.R., 2004. Model selection and model averaging in phylogenetics: advantages of Akaike Information Criterion and
Bayesian approaches over Likelihood Ratio Tests. Syst. Biol. 53, 793808.

Richards, C.M., 1982. The alteration of chromatophore expression by sex hormones in the Kenyan reed frog, Hyperolius viridiflavus. Gen. Comp. Endocrinol. 46, 5967.

Richards, C.M., Moore, W.S., 1996. A phylogeny for the African treefrog family Hyperoliidae based on mitochondrial rDNA. Mol. Phylogenet. Evol. 5, 522532.

Richards, C.L., 2006. Has the evolution of complexity in the amphibian papilla influenced anuran speciation rates? J. Evol. Biol. 19, 1222 1230.

Ryan, M.J., 1986. Factors influencing the evolution of acoustic commu nication: biological constraints. Brain, Behav. Evol. 28, 7082.

Salzburger, W., Meyer, A., 2004. The species flocks of East African cichlid fishes: recent advances on molecular phylogenetics and population genetics. Naturwissenschaften 91, 277290.

Schmidt, K., Glaw, F., Vences, M., 2001. Die Madagassischen Riedfro sche der Gattung Heterixalus. Reptilia 6, 7178.

Schiøtz, A., 1999. Treefrogs of Africa. Edition Chimaira, Frankfurt.

Summers, K., Cronin, T.W., Kennedy, T., 2003. Variation in spectral reflectance among populations of Dendrobates pumilio, the strawberry poison frog, in the Bocas del Toro Archipelago, Panama. J. Biogeogr. 30,3553 .

Swofford, D.L., 2001. PAUP*: phylogenetic analysis using parsimony (*and other methods), Version 4.0.b.10. Sinauer, Sunderland.

Van der Meijden, A., Vences, M., Hoegg, S.I., Meyer, A., 2005. A previously unrecognized radiation of ranid frogs in Southern Africa revealed by nuclear and mitochondrial DNA sequences. Mol. Phylo genet. Evol. 3, 674685 .

Vences, M., Thomas, M., Bonett, R.M., Vieites, D.R., 2005. Deciphering amphibian diversity through DNA barcoding: chances and challenges. Phil. Trans. R. Soc. Ser. B 360, 18591868.

Vences, M., Chiari, Y., Raharivololoniaina, L., Meyer, A., 2004. High mitochondrial diversity within and among populations of Malagasy poison frogs. Mol. Phylogenet. Evol. 30, 295307.

Vences, M., Glaw, F., Jesu, R., Schimmenti, G., 2000. A new species of Heterixalus (Amphibia: Hyperoliidae) from western Madagascar. Afr. Zool. 35, 269276.

Vences, M., Kosuch, J., Boistel, R., Haddad, C.F.B., La Marca, E., Lotters, S., Veith, M., 2003a. Convergent evolution of aposematic coloration in Neotropical poison frogs: a molecular phylogenetic perspective. Org. Divers. Evol. 3, 215226.

Vences, M., Kosuch, J., Glaw, F., Bohme, W., Veith, M., 2003 b. Molecular phylogeny of hyperoliid treefrogs: biogeographic origin of Malagasy and Seychellean taxa and re analysis of familial paraphyly. J. Zool. Syst. Evol Res. 41, 205215.

Vences, M., Vieites, D.R., Glaw, F., Brinkmann, H., Kosuch, J., Veith, M., Meyer, A., 2003c. Multiple overseas dispersal in amphibians. Proc. R. Soc. Lond. B 270, 24352442. 